\title{
Analyses of the effects of persistent subretinal fluid on visual/anatomic outcomes according to the type of macular neovascularization during the relaxed treat- and-extend protocol in age-related macular degeneration patients
}

Kyung Tae Kim, Ju Byung Chae, Seungheon Lee, Eoi Jong Seo and Dong Yoon Kim*

\begin{abstract}
Background: To analyze the long-term effects of persistent subretinal fluid (SRF) on visual/anatomic outcomes according to the type of macular neovascularization (MNV) during relaxed treat-and-extend regimen with antivascular endothelial growth factor (anti-VEGF) agents in age-related macular degeneration (AMD) patients.

Methods: Patients with fovea-involving type 1 or type $2 \mathrm{MNV}$, treated with a relaxed treat-and-extend regimen for 2 years were retrospectively reviewed. Eyes with SRF observed more than three times per year were defined as the 'persistent SRF (+) group'. To exclude the effects of IRF as much as possible, the eyes with persistent IRF were excluded. The effects of persistent SRF on the best-corrected visual acuity (BCVA), central subfield retinal thickness (CST), and changes in the photoreceptor layer (PRL) thickness and outer retinal bands (external limiting membrane, ellipsoid zone, and cone outer segment tip line) after anti-VEGF injection were analyzed for each MNV type.

Results: Seventy-seven eyes with type 1 MNV (44 eyes with persistent SRF) and 53 eyes with type 2 MNV (18 eyes with persistent SRF) were enrolled. Following a relaxed treat-and-extend regimen with anti-VEGF agents, BCVA and CST improved for each MNV type. In comparison between persistent SRF (+) and persistent SRF (-) group, there were no differences in the amount of change in BCVA and CST between the two groups for each MNV type during 2-year follow-up periods. In addition, there were no differences in the amount of reduction in PRL thickness and state of the outer retinal bands between the two groups for each MNV type.
\end{abstract}

Conclusions: Using a relaxed treat-and-extend regimen with anti-VEGF agents, persistent SRF did not have additional effects on visual and anatomic outcomes by 2 years, regardless of the MNV type.

Keywords: Anti-vascular endothelial growth factor, Macular neovascularization, Exudative age-related macular degeneration, Subretinal fluid

\footnotetext{
* Correspondence: umlover9@gmail.com

Department of Ophthalmology, Chungbuk National University Hospital, College of Medicine, Chungbuk National University, 776, Sunhwan-1-Ro, Seowon-Gu, Cheongju 28644, South Korea
}

(c) The Author(s). 2021 Open Access This article is licensed under a Creative Commons Attribution 4.0 International License, which permits use, sharing, adaptation, distribution and reproduction in any medium or format, as long as you give appropriate credit to the original author(s) and the source, provide a link to the Creative Commons licence, and indicate if changes were made. The images or other third party material in this article are included in the article's Creative Commons licence, unless indicated otherwise in a credit line to the material. If material is not included in the article's Creative Commons licence and your intended use is not permitted by statutory regulation or exceeds the permitted use, you will need to obtain permission directly from the copyright holder. To view a copy of this licence, visit http://creativecommons.org/licenses/by/4.0/ The Creative Commons Public Domain Dedication waiver (http://creativecommons.org/publicdomain/zero/1.0/) applies to the data made available in this article, unless otherwise stated in a credit line to the data. 


\section{Summary}

We analyzed whether persistent SRF affects to visual/ anatomic outcomes of exudative AMD according to the MNV type during relaxed treat-and-extend regimen with anti-VEGF agents. We found that small amount of persistent SRF can be tolerated without compromising visual and anatomical outcomes for 2 years, regardless of MNV type.

\section{Introduction}

Optical coherence tomography (OCT) has become a valuable noninvasive retinal imaging modality that provides useful parameters for diagnosis and follow-up monitoring in treatment of patients with exudative agerelated macular degeneration (AMD) [1]. Among the various parameters of $\mathrm{OCT}$, the presence of subretinal fluid (SRF) is widely used as a marker of active neovascularization in AMD [2, 3]. Therefore, achieving a completely dry retina was considered as a goal for the treatment of AMD. In addition, the presence of SRF on OCT was used as one of several criteria for retreatment in several large-scale randomized clinical trials $[2,4-6]$. Even in clinical practice, treatment decisions for treatand-extend or pro re nata (PRN) regimen are often driven by the presence of fluid on OCT to reduce the number of anti-vascular endothelial growth factor (antiVEGF) injections [7].

However, several studies have suggested that resolving SRF completely is not always associated with better visual prognosis [6, 8]. In the Comparison of Age-Related Macular Degeneration Treatments Trials (CATT) study, the greater proportion of patients who achieved a dry retina was not correlated with the proportion of patients who gained visual acuity improvement of more than 15 letters [8]. Another randomized clinical trial reported that there was a minimal difference between the proportions of patients who had visual acuity improvement of more than 15 letters despite the difference in the proportions of patients achieving dry retina [6]. These results question whether it is always necessary to resolve SRF completely in retina when treating AMD.

Several studies have even suggested that SRF may be associated with a better visual prognosis. One study reported that patients with SRF had better visual acuity benefits from anti-VEGF treatment $[9,10]$, and another study reported that eyes with SRF are less likely to develop retinal pigment epithelium (RPE) atrophy even under intensive anti-VEGF treatment [11]. In addition, a recent study reported that patients treated with a treatand-extend regimen who tolerated some SRF achieved good visual acuity that is comparable with that achieved when treatment aimed to resolve all SRF completely [12].
Regarding macular neovascularization (MNV) type and its association with visual prognosis, several studies have reported that minimally classic and classic lesions were associated with poorer visual outcomes, and they required more injections of anti-VEGF than occult lesions $[13,14]$. These results suggest that the visual prognosis of exudative AMD after anti-VEGF treatment may vary with MNV types. In addition, in our previous study on fibrovascular pigment epithelium detachment (PED) presenting with MNV, we reported that there were no significant differences in the visual and anatomic outcomes, regardless of the presence of persistent SRF, in AMD with type $1 \mathrm{MNV}$ using the relaxed treat-andextend regimen with anti-VEGF agents for 2 years [15]. From the results of these studies, we have questioned whether the effect of persistent SRF on the visual and anatomic outcomes of AMD is different depending on the MNV type. Therefore, in this study, we aimed to investigate the long-term effect of persistent SRF on visual and anatomic outcomes of AMD patients according to MNV types during a relaxed treat-and-extend regimen with anti-VEGF agents.

\section{Methods}

The records of patients who received an intravitreal anti-VEGF injection for exudative AMD at Chungbuk National University Hospital in Korea between January 2016 and September 2018 were analyzed retrospectively. Approval from the institutional review board and ethics committees of Chungbuk National University Hospital (No. 2020-12-018) was obtained before the initiation of the study, which was performed in compliance with the tenets of the Declaration of Helsinki. We did not obtain patient consent, since data were analyzed anonymously. The institutional review board and ethics committees of Chungbuk National University Hospital (No. 2020-12018) waived the need for informed consent.

\section{Inclusion and exclusion criteria}

Treatment naïve AMD patients with fovea-involving type 1 or $2 \mathrm{MNV}$ who were treated with a relaxed treatand-extend regimen with anti-VEGF agents and followed-up for at least 2 years were included in this study. MNV was diagnosed and classified based on fluorescein angiography (FA), indocyanine green angiography (ICGA) and spectral-domain OCT (SD-OCT). We classified MNVs into type 1 and type 2 according to published disease definitions [16]. MNVs containing both type 1 and type 2 lesions were classified as type 2 [17]. The eyes with polypoidal choroidal vasculopathy (PCV) was included in the type $1 \mathrm{MNV}$ group. Patients received three consecutive monthly intravitreal antiVEGF injections followed by a relaxed treat-and-extend regimen, allowing treatment extension by 2 weeks 
depending on disease activity (up to a maximum extension interval of 12 weeks) [18]. The disease activity during anti-VEGF treatment was defined as follow (1) a loss of BCVA of 5 letters of more than the best BCVA recorded since baseline, (2) new retinal hemorrhage, (3) presence of fluid on SD-OCT, or (4) a combination of the aforementioned. Fluid was defined as the presence of any intraretinal fluid (IRF) (resulting from disease activity as judged by the retinal specialists) or any SRF of more than $200 \mu \mathrm{m}$ in height at the foveal center. Subfoveal SRF with a height of $200 \mu \mathrm{m}$ or less or any SRF elsewhere was tolerated, and it did not prohibit extension [12]. The exclusion criteria included type $3 \mathrm{MNV}$, geographic atrophy (GA) or fibrovascular scar at the macula, and any history of photodynamic therapy or macular laser therapy. To exclude the effects of IRF as much as possible, the eyes with persistent IRF were excluded. Other ocular conditions that could compromise visual acuity or affect image quality were also excluded.

\section{Definition of 'persistent SRF'}

SRF observed more than three times per year was defined as 'persistent SRF' [3]. 'Persistent IRF' was also defined in the same way. The patients were divided into two groups according to the presence of persistent SRF for each MNV type. Eyes with persistent SRF during anti-VEGF therapy were allocated to the 'persistent SRF $(+)$ group' and the others were allocated to the 'persistent SRF (-) group'. The two groups were compared to investigate whether persistent SRF affects visual or anatomic outcomes for each MNV type.

\section{Outcome measurement}

At the initial visit, all patients underwent a comprehensive ophthalmic examination, including BCVA assessment using the Snellen chart, intraocular pressure (IOP) measurement, slit-lamp examination, color fundus photography, FA, ICGA and SD-OCT (Spectralis; Heidelberg Engineering, Heidelberg, Germany). At each subsequent visit, patients underwent ophthalmic examinations, including the assessment of BCVA, applanation tonometry, slit-lamp examination, dilated fundus examination, fundus photography, and SD-OCT [15].

The BCVA and CST of the persistent SRF (+) and persistent SRF (-) groups before the anti-VEGF injections, at 1 month after initial three loading injections (4 months), 6, 12, 18, and 24 months after the injections were compared for each MNV type. In addition, the number of intravitreal anti-VEGF injections, choroidal thickness, and outer retinal changes such as changes in the photoreceptor layer (PRL), external limiting membrane (ELM), ellipsoid zone (EZ), and cone outer segment tip (COST) line in the two groups after the injections for 2 years were compared for each MNV type [15].

\section{SD-OCT analysis}

To analyze the anatomic outcomes, the central subfield thickness (CST), subfoveal choroidal thickness (SFCT), PRL thickness, and outer retina bands (ELM, EZ, and COST line) were investigated by SD-OCT. The protocol for images of SD-OCT followed the previously introduced method, which is as follows [19]. The volumetric scans of Spectralis SD-OCT were acquired with Spectralis Viewing Module (Version 6.0.9.0). For images obtained by Spectralis SD-OCT, a custom $20^{\circ} \times 20^{\circ}$ volume acquisition protocol which covered $6 \mathrm{~mm} \times 6 \mathrm{~mm}$ surface of the macula was used to obtain one set of highspeed scans form each eye. With this protocol, 49 crosssectional B-scan images were obtained, each composed of 512 A-scans. The integrated follow-up mode of the device was used to ensure that the exact same retinal area was imaged at every follow-up visit [19]. The PRL thickness was measured as the distance from the outer margin of the outer plexiform layer (OPL) to the anterior margin of the RPE [20]. If there was SRF at the fovea, the PRL thickness was measured as the distance from the outer margin of the OPL to the outer end of the photoreceptors [21]. Segmenting the outer margin of the OPL and the anterior margin of the RPE on SD-OCT image was manually performed using the segmentation software built into SD-OCT by two different retinal specialists (JBC and EJS) who were masked to the study design. The PRL thickness was measured automatically after the segmentation through the built-in software. The values of PRL thickness at 3 locations, such as fovea center and $500 \mu \mathrm{m}$ away from fovea center, were averaged (Fig. 1). The average of both measurements from each retinal specialist was used for the analysis.

\section{Statistical analysis}

Data are presented as the mean \pm SD or numbers (\%). The Kolmogorov-Smirnov test was used for assessing normality. The statistical analyses were conducted using SPSS version 22.0 software (SPSS, Inc., Chicago, IL, USA). A $P$-value $<0.05$ was considered statistically significant.

The paired $t$-test was used to evaluate the differences in BCVA and CST between the baseline and last visit. And the Student's t-test was used to evaluate the differences in parameters including BCVA, CST, PRL thickness and the number of injections between the 'persistent SRF (+) group' and 'persistent SRF (-) group' for each CNV type. The differences in the status of the ELM, EZ, and COST line between groups was analyzed by Pearson's $\chi^{2}$ test. 

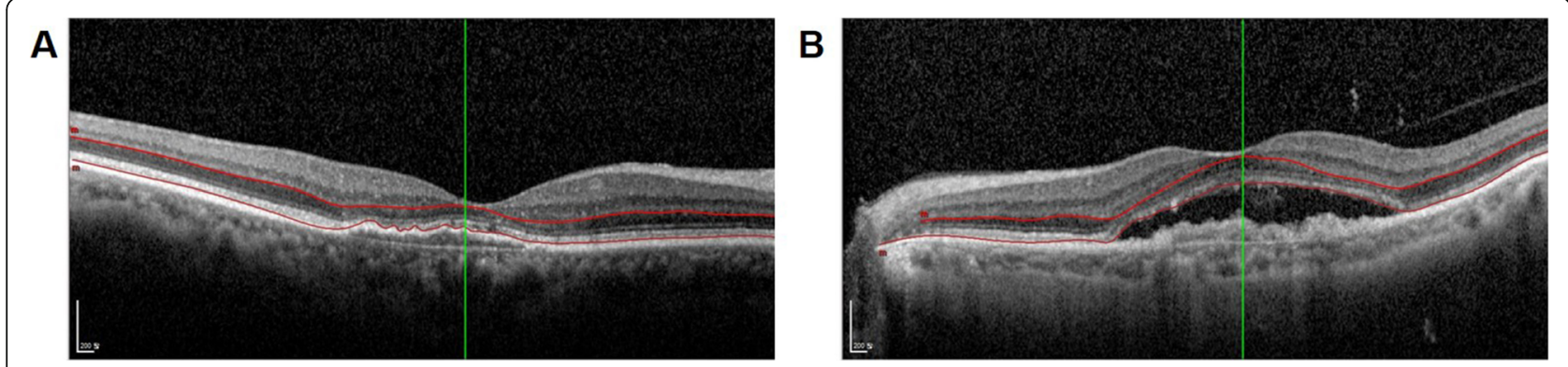

Fig. 1 Segmentation the retinal layer and measuring the photoreceptor layer (PRL) thickness. Segmenting the anterior margin of the retinal pigment epithelium (RPE) and the outer margin of the outer plexiform layer (OPL) was manually performed using the segmentation software. The PRL thickness was measured automatically after the segmentation through the software built into SD-OCT. The PRL thickness was measured as the distance from the outer margin of the OPL to the anterior margin of the RPE in eyes without SRF (A). In eyes with SRF, the PRL thickness was measured as the distance from the outer margin of the OPL to the outer end of the photoreceptors (B)

\section{Results}

A total of 77 eyes from 77 patients with type $1 \mathrm{MNV}$ and 53 eyes from 53 patients with type $2 \mathrm{MNV}$ were included in this study (12 eyes from type $2 \mathrm{CNV}$ group were excluded because persistent IRF was observed). Among 77 eyes with type $1 \mathrm{MNV}, 28$ eyes with PCV were included. The demographics and baseline ocular findings of the patients are summarized in Table 1. The mean ages of patients were $74.2 \pm 7.2$ years for type 1 MNV group and $76.6 \pm 7.3$ years for type $2 \mathrm{MNV}$ group. The mean baseline BCVA of type $1 \mathrm{MNV}$ group was better than that of type $2 \mathrm{MNV}$ group $(0.54 \pm 0.30$ in

Table 1 Patient demographics and baseline ocular findings in type1 CNV and type 2 CNV group

\begin{tabular}{|c|c|c|c|}
\hline Characteristics. & Type 1 CNV group $(n=77)$ & Type 2 CNV group $(n=53)$ & $p$-value \\
\hline Age, years (mean $\pm S D$ ) & $74.2 \pm 7.2$ & $76.6 \pm 7.3$ & 0.067 \\
\hline Sex, male/female (\%) & $51 / 26(66 / 34)$ & $29 / 24(55 / 45)$ & 0.072 \\
\hline Lens status, phakic/pseudophakic (\%) & $57 / 20(74 / 26)$ & $33 / 20(62 / 38)$ & 0.153 \\
\hline Best-corrected visual acuity, logMAR (mean \pm SD) & $0.54 \pm 0.30$ & $0.75 \pm 0.36$ & 0.001 \\
\hline Central subfield retinal thickness, $\mu \mathrm{m}$ (mean \pm SD) & $398.06 \pm 123.07$ & $436.74 \pm 131.05$ & 0.089 \\
\hline Subfoveal choroidal thickness, $\mu \mathrm{m}$ (mean \pm SD) & $293.64 \pm 79.97$ & $229.32 \pm 76.76$ & $<0.001$ \\
\hline Anti-VEGF, n (\%) & & & 0.324 \\
\hline Bevacizumab & $10(13)$ & $5(9)$ & \\
\hline Ranibizumab & $12(15)$ & $12(23)$ & \\
\hline Aflibercept & $39(51)$ & $20(38)$ & \\
\hline Mixed & $16(21)$ & $16(30)$ & \\
\hline \multicolumn{4}{|l|}{ No. of intravitreal anti-VEGF injections (mean \pm SD) } \\
\hline 1 year & $7.0 \pm 1.6$ & $6.8 \pm 1.4$ & 0.436 \\
\hline 2 year & $5.1 \pm 1.9$ & $5.1 \pm 1.4$ & 0.940 \\
\hline Total & $12.0 \pm 3.0$ & $11.8 \pm 2.4$ & 0.631 \\
\hline \multicolumn{4}{|l|}{ Fluid on OCT at baseline, n (\%) } \\
\hline SRF & $77(100)$ & $49(92)$ & 0.014 \\
\hline IRF & $2(3)$ & $39(74)$ & $<0.001$ \\
\hline
\end{tabular}

$C N V$ choroidal neovascularization, $S D$ standard deviation, $\log M A R$ logarithm of the minimal angle of resolution, VEGF vascular endothelial growth factor, OCT optical coherence tomography 
type $1 \mathrm{MNV}$ group, $0.75 \pm 0.36$ in type $2 \mathrm{MNV}$ group, $p=0.001$ ), and the mean baseline SFCT of type $2 \mathrm{MNV}$ group was lower than that of type $1 \mathrm{MNV}$ group (293.64 \pm 79.97 in type $1 \mathrm{MNV}$ group, $229.32 \pm 76.76$ in type $2 \mathrm{MNV}$ group, $p<0.001$ ). Bevacizumab in 13 eyes, ranibizumab in 17 eyes, and aflibercept in 47 eyes were used for the initial loading treatment in the type $1 \mathrm{MNV}$ group. In the type $2 \mathrm{MNV}$ group, bevacizumab in 5 eyes, ranibizumab in 20 eyes, and aflibercept in 28 eyes were used for the initial loading treatment. In each group, 16 eyes were treated with two or more types of anti-VEGF after the initial loading treatment. The prevalence of SRF or IRF at baseline significantly differed in the two groups (77 eyes with SRF in type $1 \mathrm{MNV}, 49$ eyes with SRF in type $2 \mathrm{MNV}, p=0.014 ; 2$ eyes with IRF in type 1 MNV, 39 eyes with IRF in type $2 \mathrm{MNV}, p<0.001$ ). There were no significant differences in the mean baseline CST and the number of intravitreal anti-VEGF injections between the two groups.

\section{Comparison of the persistent SRF (+) and persistent SRF (-) groups for each MNV type}

We divided the eyes into two groups according to the presence of persistent SRF in type $1 \mathrm{MNV}$ and type 2 MNV groups. There were 44 eyes in the persistent SRF (+) group and 33 eyes in the persistent SRF (-) group in the type $1 \mathrm{MNV}$ group. The number of intravitreal anti-
VEGF injections during the follow-up did not differ in the two groups in the type $1 \mathrm{MNV}$ group. In addition, there were no significant differences in the BCVA, CST, SFCT, the state of outer retinal bands and the mean change of PRL thickness between the two groups for 2 years (Table 2). In type $1 \mathrm{MNV}$ group, analysis of PED status was performed. The heights of PED in the persistent SRF (+) and persistent SRF (-) group were $175.80 \pm$ 104.82 and $196.25 \pm 105.81$, respectively $(p=0.403)$. In the persistent SRF (-) group, the complete regression of PED was observed in 3 eyes, and there were no eyes with complete regression of PED in the persistent SRF $(+)$ group. The amounts of changes of PED height in the persistent SRF $(+)$ and persistent SRF (-) group were $32.62 \pm 71.15$ and $-87.66 \pm 93.68$, respectively $(p=$ $0.007)$.

In type $2 \mathrm{MNV}$ group, 18 eyes were classified into the persistent SRF (+) group whereas 35 eyes were classified into the persistent SRF (-) group. The number of intravitreal anti-VEGF injections administered during the follow-up did not differ in the two groups. The mean SFCT in the persistent SRF (+) group was significantly higher than that in the persistent SRF (-) group at baseline and after 2 years. In addition, the mean CST in the persistent SRF (+) group was higher than that in the persistent SRF (-) group. However, there were no significant differences in the BCVA, the state of outer retinal

Table 2 Visual/anatomic outcomes between 'persistent SRF (+) group' and 'persistent SRF (-) group' in type 1 CNV and type 2 CNV

\begin{tabular}{|c|c|c|c|c|c|c|}
\hline \multirow[t]{2}{*}{ Characteristics. } & \multicolumn{2}{|l|}{ Type 1 CNV } & \multirow[t]{2}{*}{$p$} & \multicolumn{2}{|l|}{ Type 2 CNV } & \multirow[t]{2}{*}{$p$} \\
\hline & $\begin{array}{l}\text { Persistent SRF (+) } \\
\text { group }(n=44)\end{array}$ & $\begin{array}{l}\text { Persistent SRF (-) } \\
\text { group }(n=33)\end{array}$ & & $\begin{array}{l}\text { Persistent SRF }(+) \\
\text { group }(n=18)\end{array}$ & $\begin{array}{l}\text { Persistent SRF (-) } \\
\text { group }(n=35)\end{array}$ & \\
\hline \multicolumn{7}{|c|}{ No. of intravitreal anti-VEGF injections (mean \pm SD) } \\
\hline 1 year & $7.2 \pm 1.5$ & $6.7 \pm 1.7$ & 0.175 & $7.3 \pm 1.4$ & $6.5 \pm 1.4$ & 0.059 \\
\hline 2 year & $5.4 \pm 1.7$ & $4.7 \pm 2.0$ & 0.092 & $5.4 \pm 1.8$ & $4.9 \pm 1.1$ & 0.276 \\
\hline Total & $12.6 \pm 2.7$ & $11.3 \pm 3.3$ & 0.073 & $12.7 \pm 2.7$ & $11.4 \pm 2.1$ & 0.061 \\
\hline \multicolumn{7}{|l|}{ Values at baseline (mean $\pm S D)$} \\
\hline$B C V A, \log M A R$ & $0.52 \pm 0.27$ & $0.58 \pm 0.35$ & 0.453 & $0.70 \pm 0.30$ & $0.78 \pm 0.39$ & 0.443 \\
\hline CST, $\mu \mathrm{m}$ & $386.95 \pm 119.15$ & $412.88 \pm 128.44$ & 0.364 & $446.11 \pm 121.83$ & $431.91 \pm 137.02$ & 0.713 \\
\hline $\mathrm{SFCT}, \mu \mathrm{m}$ & $305.03 \pm 78.74$ & $276.78 \pm 72.40$ & 0.142 & $264.31 \pm 76.03$ & $211.26 \pm 71.79$ & 0.023 \\
\hline \multicolumn{7}{|c|}{ Values at 2 years after anti-VEGF injections (mean \pm SD) } \\
\hline$B C V A, \log M A R$ & $0.35 \pm 0.25$ & $0.35 \pm 0.26$ & 0.900 & $0.42 \pm 0.28$ & $0.55 \pm 0.35$ & 0.192 \\
\hline CST, $\mu \mathrm{m}$ & $303.02 \pm 57.34$ & $275.45 \pm 67.67$ & 0.057 & $314.28 \pm 67.56$ & $269.11 \pm 53.84$ & 0.011 \\
\hline $\mathrm{SFCT}, \mu \mathrm{m}$ & $294.14 \pm 77.97$ & $262.64 \pm 73.66$ & 0.077 & $249.94 \pm 77.36$ & $200.46 \pm 75.45$ & 0.029 \\
\hline ELM intact/defect & $41 / 3$ & $29 / 4$ & 0.423 & $14 / 4$ & $19 / 16$ & 0.095 \\
\hline EZ intact/defect & $23 / 21$ & $19 / 14$ & 0.644 & $8 / 10$ & $8 / 27$ & 0.105 \\
\hline COST line intact/defect & $1 / 43$ & $2 / 31$ & 0.395 & $1 / 17$ & $0 / 35$ & 0.159 \\
\hline $\begin{array}{l}\triangle P R L \text { thickness from After } \\
\text { 3rd injection }\end{array}$ & $-5.70 \pm 2.66$ & $-6.58 \pm 2.45$ & 0.145 & $-6.22 \pm 3.72$ & $-8.40 \pm 3.84$ & 0.054 \\
\hline
\end{tabular}

CNV choroidal neovascularization, SRF subretinal fluid, VEGF vascular endothelial growth factor, SD standard deviation, BCVA best-corrected visual acuity, logMAR logarithm of the minimal angle of resolution, CST central subfield thickness, SFCT subfoveal choroidal thickness, ELM external limiting membrane, EZ ellipsoid zone, COST con outer segment tip, PRL photoreceptor layer 
bands, and the mean change of PRL thickness between the two groups for 2 years (Table 2).

\section{Changes in BCVA and CST}

With the treat-and-extend treatment strategy, the BCVA and CST were significantly improved and maintained in both persistent SRF $(+)$ and persistent SRF $(-)$ groups for each MNV type. For the type $1 \mathrm{MNV}$ group, the BCVAs of the persistent SRF (+) and persistent SRF (-) groups were improved to $0.35 \pm 0.25(p<0.001)$ and $0.35 \pm 0.26(p<0.001)$, respectively, from the baseline after 2 years. The CST was improved to $303.02 \pm 57.34$ in the persistent SRF $(+)$ group $(p<0.001)$ and $275.45 \pm$ 67.67 in the persistent SRF $(-)$ group $(p<0.001)$ after 2 years. For the type $2 \mathrm{MNV}$ group, the BCVAs of the persistent SRF $(+)$ and persistent SRF $(-)$ groups were improved to $0.42 \pm 0.28(p<0.001)$ and $0.55 \pm 0.35(p<$ 0.001) from baseline after 2 years, respectively. The CST after 2 years was improved to $314.28 \pm 67.56$ in the persistent SRF $(+)$ group $(p<0.001)$ and $269.11 \pm 53.84$ in the persistent SRF $(-)$ group $(p<0.001)$. The BCVA and CST changes from baseline was presented in Fig. 2. The amount of BCVA and CST changes during the-2year follow-up did not differ between the persistent SRF $(+)$ and persistent SRF (-) groups in the type 1 groups (BCVA, $p=0.756$; CST, $p=0.085$ ). In the type $2 \mathrm{MNV}$ group, the amount of BCVA and CST changes during the-2-year follow-up did not differ between the persistent SRF (+) and persistent SRF (-) groups (BCVA, $p=$ 0.532; CST, $p=0.407$ ).

\section{Changes in PRL thickness}

During the follow-up, the PRL thickness continuously decreased in the persistent SRF $(+)$ and persistent SRF

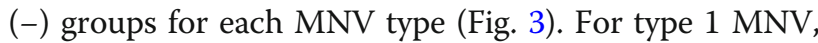
the amount of decrease in PRL thickness during the-2year follow-up was not different between the persistent SRF $(+)$ and persistent SRF $(-)$ groups $(p=0.145)$. For
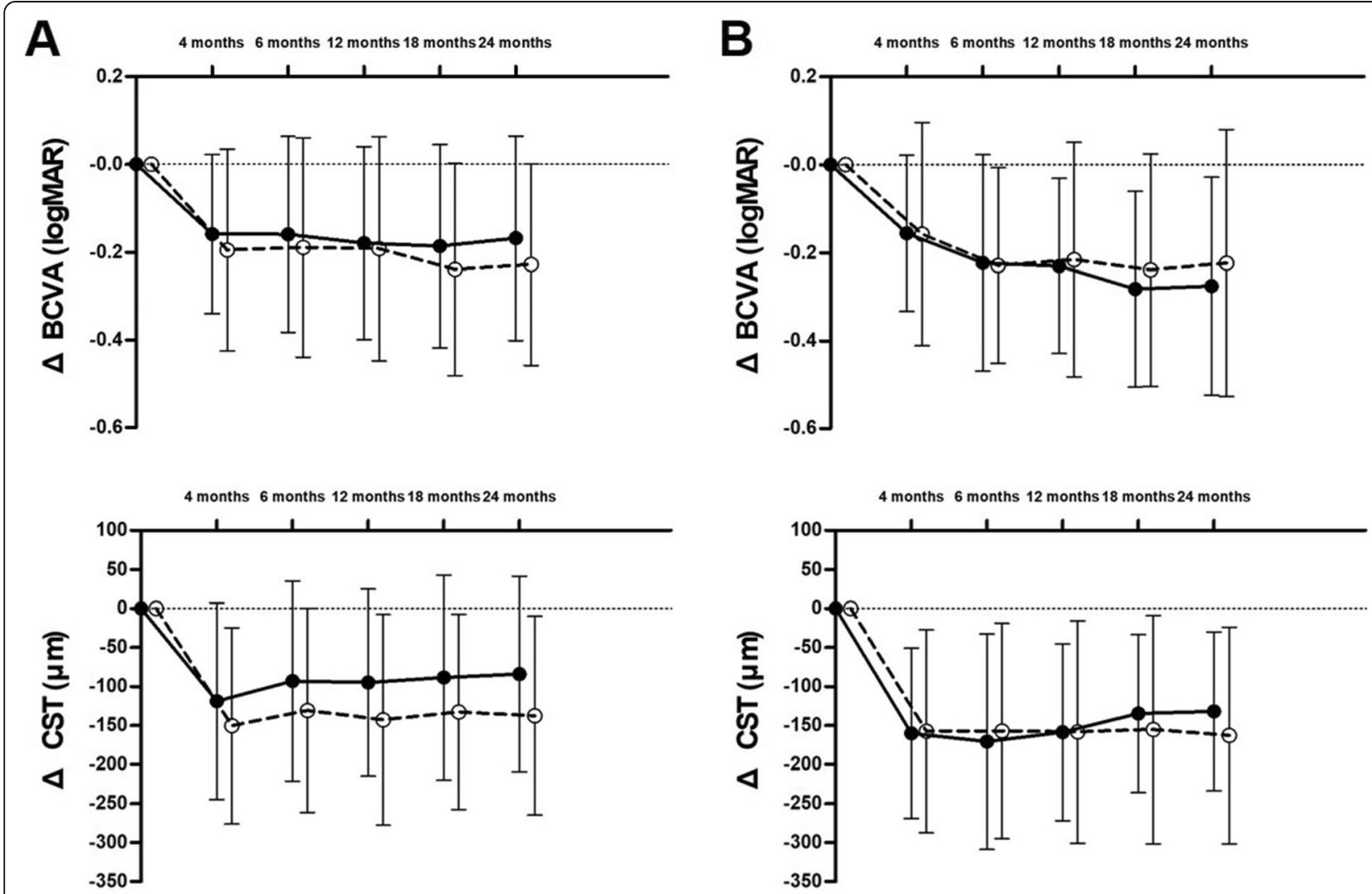

\section{$\rightarrow$ Persistent SRF (+) group \\ $-\Theta$. Persistent SRF (-) group}

Fig. 2 Graph illustrating changes in the amount of decrease in best-corrected visual acuity (BCVA) and central subfield retinal thickness (CST). Both type 1 MNV (A) and type 2 MNV (B) groups show significant improvement in the BCVA and CST after 2 years, regardless of the presence of persistent SRF. The BCVA and CST changes from baseline with the relaxed treat-and-extend regimen did not differ in the persistent SRF (+) and persistent SRF (-) groups for both type 1 and type 2 MNV during the 2-year follow-up 


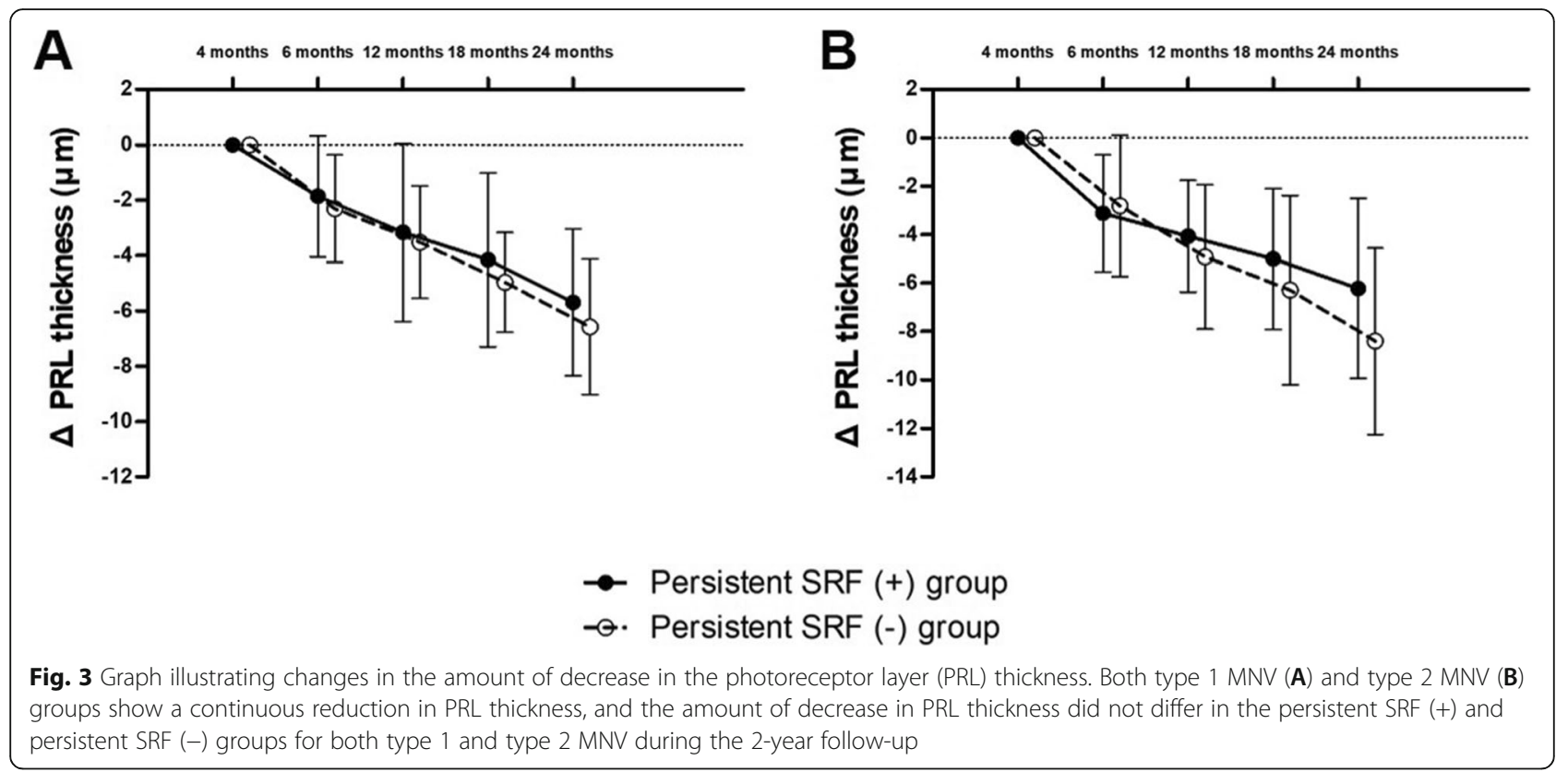

type $2 \mathrm{MNV}$, the slope of the decrease in PRL thickness in the persistent SRF (-) group tended to be steeper than that of the persistent SRF (+) group. However, the amount of decrease in PRL thickness was not different between the two groups during the 2-year follow-up $(p=0.054)$. Representative cases with persistent SRF from type 1 and type $2 \mathrm{MNV}$ groups are shown in Fig. 4.

\section{Sub-analysis of eyes with PCV in type $1 \mathrm{MNV}$ group}

Among 77 eyes with type $1 \mathrm{MNV}, 28$ eyes with PCV were included (17 eyes in the persistent SRF $(+)$ group and 11 eyes in the persistent SRF (-) group). The total number of intravitreal anti-VEGF injections administered during the follow-up was $12.8 \pm 1.6$ in the persistent SRF (+) group $(7.0 \pm 1.1$ at 1 year, $5.8 \pm 1.2$ at 2 year). In the persistent SRF (-) group, the total number of intravitreal anti-VEGF injections was $12.0 \pm 3.1(7.5 \pm 1.9$ at 1 year, $4.6 \pm 2.0$ at 2 year). There were no significant differences in the number of intravitreal anti-VEGF injections between the two groups $(p=0.452$ at 1 year, $p=0.059$ at 2 years, $p=0.428$ for 2 years).

With the treat-and-extend treatment strategy, the BCVA and CST were significantly improved and maintained in both persistent SRF $(+)$ and persistent SRF (-) groups. The BCVAs of the persistent SRF $(+)$ and persistent SRF $(-)$ group were improved $0.44 \pm 0.22(p=$ $0.034)$ and $0.33 \pm 0.30(p=0.008)$, respectively, from the baseline after 2 years. The CST was improved to $326.82 \pm 56.53$ in the persistent SRF $(+)$ group $(p=$ $0.002)$ and $273.45 \pm 75.68$ in the persistent SRF (-) group $(p=0.003)$ after 2 years. The amount of BCVA and CST changes during the-2-year follow-up did not differ between the persistent SRF (+) and persistent SRF (-) groups in the eyes with PCV (BCVA, $p=0.251$; CST, $p=0.861$.

During the follow-up, the PRL thickness continuously decreased in the persistent SRF (+) and persistent SRF (-) groups. The amounts of decrease in PRL thickness during the-2-year follow-up were $-6.41 \pm 1.46$ and $6.64 \pm 2.69$ in the persistent SRF $(+)$ and persistent SRF $(-)$ groups, respectively. There was no significant difference in the amount of decrease in PRL thickness during the 2-year follow-up $(p=0.804)$.

\section{Discussion}

In this study, we investigated the effects of persistent SRF on the visual and anatomic outcomes of eyes with exudative AMD according to the MNV type. The primary finding was that BCVA and CST were improved and well maintained for 2 years in both type 1 and type $2 \mathrm{MNV}$ groups using the relaxed treat-and-extend regimen with anti-VEGF agents, regardless of the presence of persistent SRF. The second finding was that the number of intravitreal anti-VEGF injections in the persistent SRF (+) and persistent SRF (-) groups did not differ with the relaxed treat-and-extend regimen. Furthermore, anatomical changes in the outer retina, including changes in the outer retinal bans and PRL thickness, were not different in both MNV groups, regardless of the presence of persistent SRF.

The presence of fluid on OCT is used widely as a marker of activity of the neovascular process [2, 3]. In line with this, several studies have reported the negative impact of SRF on visual outcome. Hoerster et al. reported that SRF correlated significantly with impaired 


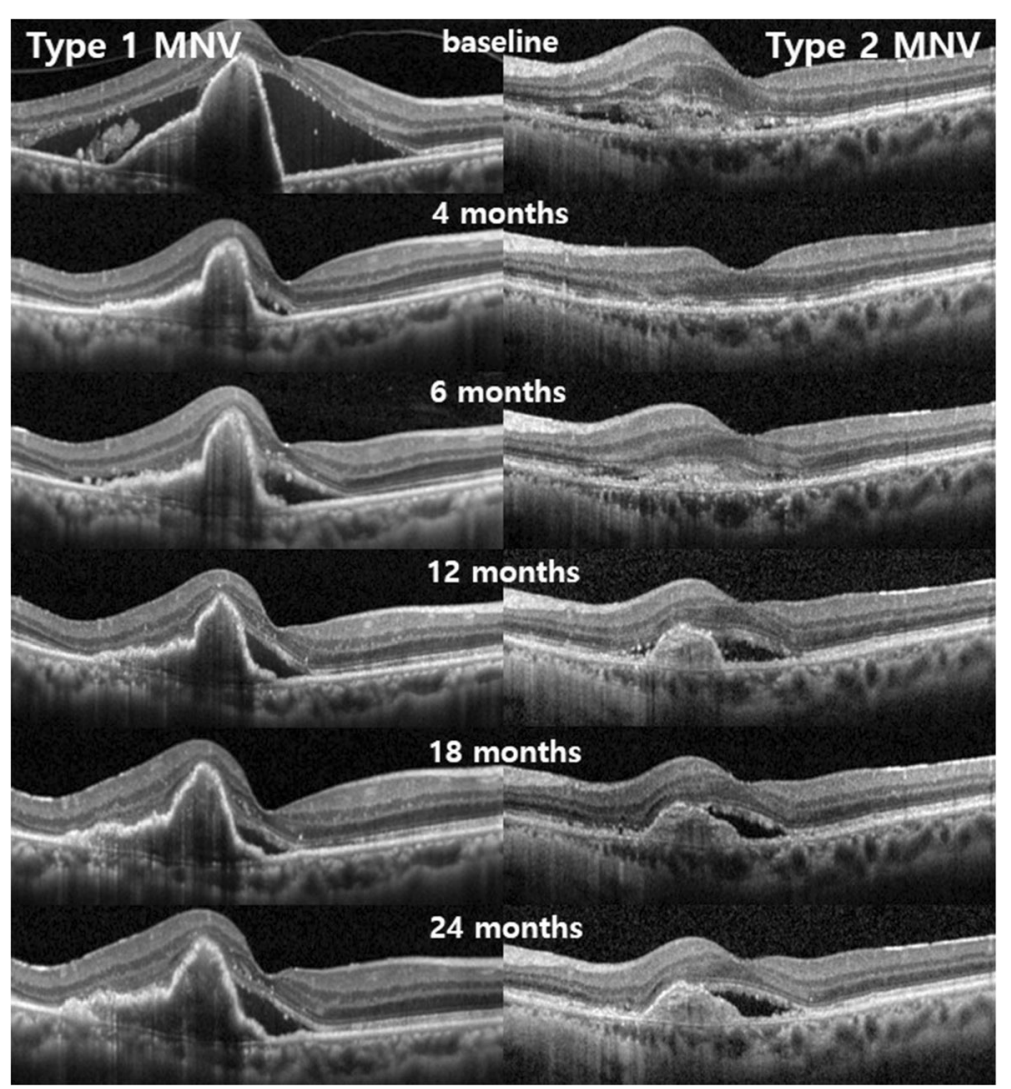

Fig. 4 Optical coherence tomography (OCT) images of the representative cases with persistent SRF from the type 1 and type 2 MNV groups. The first case (left column) is of a 74-year-old male with type 1 MNV treated using the treat-and-extend regimen with aflibercept during the 2-year follow-up. The Snellen BCVA was 0.3, and fibrovascular PED and SRF were observed on SD-OCT at baseline. A total 13 times of intravitreal aflibercept injection were administered for 2 years. Although small amount of SRF persisted for 2 years, the Snellen BCVA was improved to 0.5 and apparent atrophic or degenerative change in the outer retina were not observed for 2 years. The second case (right column) is of a 68 -yearold female with type $2 \mathrm{MNV}$ treated using the treat-and-extend regimen with aflibercept during the 2-year follow-up. The Snellen BCVA was 0.5, and subretinal MNV, SRF and IRF were observed on SD-OCT at baseline. A total 12 times of intravitreal aflibercept injection were administered for 2 years. The SRF and IRF were subsided after 3-monthly aflibercept intravitreal injections, but SRF recurred at 6 months and persisted. Although persistent SRF was observed, the Snellen BCVA was improved to 0.7 and external limiting membrane and ellipsoid zone were intact at 2 years

BCVA [22], and Golbaz et al. reported that SRF was more frequently associated with recurrent disease [23]. In addition, one study demonstrated that retinal alteration like SRF affect negatively to retinal sensitivity by using microperimetry [24]. On the other hand, the evidence that SRF may be associated with a positive effect on VA has recently been supported by several studies. Some studies have reported that the presence of SRF at any time is associated with higher VA levels $[9,25]$. Other studies have demonstrated that patients with SRF have better visual acuity benefits from anti-VEGF therapy $[9,10]$. In addition, Sato et al. reported that eyes with SRF are less likely to develop RPE atrophy [11]. As a possible reason for the positive effects of SRF on visual prognosis, one study suggested that SRF could be suggestive of a functional perfused neovascular net providing RPE and PRL survival in contrast to advanced vascular atrophy in the sub-RPE space [26]. In this study, we investigated the effect of persistent SRF on visual and anatomic outcomes in eyes with exudative AMD. The final BCVA and the degree of improvement in BCVA in the persistent SRF $(+)$ group were not different significantly from those in the persistent SRF (-) group. In addition, the persistent SRF had no additional effects on the outer retina with the relaxed treat-and-extend regimen during the 2-year follow-up period. In other words, the persistent SRF (+) group, compared with the persistent SRF (-) group, showed non-inferior visual and anatomic outcomes for 2 years with the relaxed treat-andextend regimen. These results suggest that persistent SRF can be tolerated without compromising visual and anatomic outcomes during exudative AMD treatment with the relaxed treat-and-extend regimen by 2 years, regardless of the MNV type. Figure 4 shows representative cases with persistent SRF from the type 1 and type 2 MNV groups. 
Unlike SRF, the negative effects of IRF on BCVA were demonstrated consistently by several studies [25, 27, 28]. Ritter et al. reported that eyes with IRF presented with the lowest initial VA, and IRF had the strongest negative predictive value for functional improvement [27]. And other studies demonstrated that eyes with IRF show reduced initial visual acuity by a mean of two lines on ETDRS charts [9, 25, 28]. Unfortunately, we could not investigate the effect of persistent IRF, because the main purpose of this study was to investigate the effect of persistent SRF for each MNV type. Therefore, we excluded the eyes with persistent IRF to minimize the effect of IRF on visual and anatomic outcomes. In addition, the eyes with persistent IRF were relatively rare because IRF caused by exudative MNV has been known that they are exquisitely responsive to anti-VEGF treatment [26]. Bolz et al. also reported an almost complete reduction of exudative IRF 1 week after a single anti-VEGF injection [29]. In current study, IRF was resolved completely after 3-monthly intravitreal anti-VEGF injection in both type 1 and type $2 \mathrm{CNV}$ groups. Persistent IRF was observed in only 12 eyes despite anti-VEGF treatment, and they were excluded from the type $2 \mathrm{MNV}$ group. Considering that RPE atrophy or scarring were observed in all these 12 eyes, the IRF observed in these eyes was considered degenerative IRF and not related to exudative MNV but neurosensory degeneration [26].

In multiple randomized trials, eyes treated with fixeddosing anti-VEGF injection had better visual acuity at 1 and 2 years than those that received less frequent injections $[2,30,31]$. However, a monthly treatment regimen may increase the overall treatment burden for patients. Therefore, to minimize the injection frequency, a proactive dosing regimen, such as the treat-and-extend regimen, was introduced [32]. Several studies reported that the treat-and-extend regimen was statistically noninferior and clinically comparable with a monthly regimen in improving VA for all three types of anti-VEGF [33-35]. Furthermore, recent randomized clinical trial used extension criteria to extend the injection interval even when a small amount of SRF was observed with the treat-and-extend regimen [12]. This study demonstrated that patients treated with a ranibizumab treat-andextend regimen who tolerated some SRF (subfoveal SRF of $200 \mu \mathrm{m}$ or less) achieved VA comparable with that achieved when treatment aimed to resolve all SRF completely [12]. In current study, a relaxed treat-and-extend regimen, which allows subfoveal SRF of $200 \mu \mathrm{m}$ or less, was used as treatment regimen, and we confirmed that the BCVA and CST were improved and well maintained for 2 years in both type 1 and type $2 \mathrm{MNV}$ groups, regardless of the persistent SRF. In addition, considering that there was no difference in the number of antiVEGF injections between the persistent SRF (+) and persistent SRF (-) groups for each MNV type, it was expected that a relaxed treat-and-extend regimen could reduce the number of anti-VEGF injections even in the presence of tolerable SRF. However, the exact amount and nature of tolerable SRF have not been established for the treat-and-extend regimen for the treatment of exudative AMD. Therefore, a prospective study is warranted to investigate the exact extent and properties of tolerable SRF for anti-VEGF treatment of exudative AMD.

In this study, the proportion of eyes with persistent SRF was higher for type $1 \mathrm{MNV}$ than for type $2 \mathrm{MNV}$. This might be explained by the varying fluid patterns with the MNV type. A study reported that SRF is the predominant form of exudation and the first typical exudative sign in type $1 \mathrm{MNV}$ lesions, and the presence of IRF in type $1 \mathrm{MNV}$ lesions presumably indicates damage to the outer blood-retinal barrier in the form of RPE dysfunction and disruption in the tight junctions that contribute to the ELM band on SD-OCT. [16] This study also reported that IRF predominates with type 2 MNV lesions rather than SRF [16]. Similarly, in current study, SRF was observed in all the eyes with type 1 MNV, and IRF was more frequently observed in eyes with type $2 \mathrm{MNV}$ than those with type $1 \mathrm{MNV}$ on the image of initial SD-OCT. In addition, the proportion of eyes with persistent SRF was different between the type 1 (57\%) and type $2 \mathrm{MNV}$ (34\%) groups.

A strength of the current study is that it is the first study to analyze the effect of persistent SRF on visual and anatomic outcomes according to MNV type. Through this analysis, we confirmed that persistent SRF did not affect the visual or anatomic outcomes additionally during anti-VEGF treatment with the relaxed treatand-extend regimen in both type 1 and type $2 \mathrm{MNV}$ lesions by 2 years. Therefore, it is considered that a favorable outcome can be achieved with a relatively small number of anti-VEGF injections even in eyes with persistent SRF regardless of MNV type. However, this study has some notable limitations that are inherent in its retrospective and nonrandomized design with a small sample size. Second, the number of anti-VEGF injections received by each patient and the drug type (bevacizumab, ranibizumab, and aflibercept) were not controlled; thus, a heterogeneous population was enrolled. Third, the eyes with PCV were included in the type $1 \mathrm{MNV}$ group, and some mixed case which have both type 1 and type $2 \mathrm{MNV}$ were included in the type $2 \mathrm{MNV}$ group. Fourth, with the exclusion of the eyes with persistent IRF despite of anti-VEGF therapy, patients with relatively mild exudative AMD may have been included in the type $2 \mathrm{MNV}$ group. Fifth, since a quantitative analysis of SRF has not been conducted, the quantitative criteria for tolerable SRF in anti-VEGF treatment have not 
been provided. Sixth, due to the relatively short-term follow-up period of 2 years, it might be difficult to determine the effects of persistent SRF on visual function, requiring long-term follow-up studies. Finally, the characterization of visual function in the included patients may have been relatively poor because objective tests for visual function were not performed. Using visual function tests such as static perimetry or multifocal ERG may facilitate more objective evaluation of patients' visual function; thereby, we would better understand the relationship between functional visual acuity and anatomical changes in AMD patients. Therefore, further prospectively designed studies with large sample sizes using objective visual function tests are warranted. In addition, quantitative and qualitative studies to investigate the criteria of tolerable SRF also be needed in the future.

In conclusion, we found that visual and anatomical prognoses were relatively good and well maintained using a relaxed treat-and-extend regimen, regardless of the presence of persistent SRF in both type 1 and type 2 MNV. And these results of this study were obtained by excluding eyes with persistent IRF. Using a relaxed treatand-extend regimen with anti-VEGF agents, persistent SRF might be tolerated without compromising visual and anatomic outcomes by 2 years, regardless of the MNV type.

\footnotetext{
Abbreviations

SRF: Subretinal fluid; MNV: Macular neovascularization; VEGF: Vascular endothelial growth factor; AMD: Age-related macular degeneration; RPE: Retinal pigment epithelium; BCVA: Best-corrected visual acuity; CST: Central subfield retinal thickness; SFCT: Subfoveal choroidal thickness; ELM: External limiting membrane; EZ: Ellipsoid zone; COST: Cone outer segment tip; PED: Pigment epithelium detachment; OPL: Outer plexiform layer
}

\section{Acknowledgments}

Not applicable.

\section{Authors' contributions}

Author contributions were as follows: design of the study (KK, JC, SL, ES and DK); collection and management of the data (KK, JC, SL, and DK); analysis and interpretation of the data (KK, JC, ES, and DK); preparation of the manuscript and statistical analysis and interpretation (KK, JC, and DK); review and approval of the manuscript (KK, JC, SL, ES, and DK).

\section{Funding}

There was no specific funding or financial support for this study.

\section{Availability of data and materials}

The data used to support the findings of this study are restricted by the Institutional Review Board of Chungbuk National University Hospital in order to protect PATIENT PRIVACY. Data are available from Dong Yoon Kim (umlover9@gmail.com) for researchers who meet the criteria for access to confidential data.

\section{Declarations}

\section{Ethics approval and consent to participate}

This retrospective study was approved by the Ethics Committee of Chungbuk National University Hospital (No. 2020-12-018). The institutional review board and ethics committees of Chungbuk National University
Hospital (No. 2020-12-018) waived the need for informed consent. The study adhered to the tenets of the Declaration of Helsinki.

\section{Consent for publication}

Not applicable.

\section{Competing interests}

The authors declare that they have no conflicts interests.

Received: 29 March 2021 Accepted: 26 July 2021

Published online: 10 August 2021

\section{References}

1. Drexler W, Fujimoto JG. State-of-the-art retinal optical coherence tomography. Prog Retin Eye Res. 2008;27(1):45-88. https://doi.org/10.1016/j. preteyeres.2007.07.005.

2. Martin DF, Maguire MG, Ying GS, Grunwald JE, Fine SL, Jaffe GJ. Ranibizumab and bevacizumab for neovascular age-related macular degeneration. N Engl J Med. 2011;364(20):1897-908. https://doi.org/10.1056/ NEJMoa1102673.

3. Wickremasinghe SS, Janakan V, Sandhu SS, Amirul-Islam FM, Abedi F, Guymer RH. Implication of recurrent or retained fluid on optical coherence tomography for visual acuity during active treatment of neovascular agerelated macular degeneration with a treat and extend protocol. Retina. 2016;36(7):1331-9

4. Holz FG, Amoaku W, Donate J, Guymer RH, Kellner U, Schlingemann RO, et al. Safety and efficacy of a flexible dosing regimen of ranibizumab in neovascular age-related macular degeneration: the SUSTAIN study. Ophthalmology. 2011;118(4):663-71. https://doi.org/10.1016/j.ophtha.201 0.12 .019 .

5. Lalwani GA, Rosenfeld PJ, Fung AE, Dubovy SR, Michels S, Feuer W, et al. A variable-dosing regimen with intravitreal ranibizumab for neovascular agerelated macular degeneration: year 2 of the PrONTO Study. Am J Ophthalmol. 2009;148(1):43-58.e1.

6. Schmidt-Erfurth U, Kaiser PK, Korobelnik JF, Brown DM, Chong V, Nguyen $\mathrm{QD}$, et al. Intravitreal aflibercept injection for neovascular age-related macular degeneration: ninety-six-week results of the VIEW studies. Ophthalmology. 2014;121(1):193-201. https://doi.org/10.1016/j.ophtha.2013. 08.011.

7. Jaffe GJ, Kaiser PK, Thompson D, Gibson A, Saroj N, Vitti R, et al. Differential response to anti-VEGF regimens in age-related macular degeneration patients with early persistent retinal fluid. Ophthalmology. 2016;123(9):185664. https://doi.org/10.1016/j.ophtha.2016.05.016.

8. Martin DF, Maguire MG, Fine SL, Ying GS, Jaffe GJ, Grunwald JE, et al. Ranibizumab and bevacizumab for treatment of neovascular age-related macular degeneration: two-year results. Ophthalmology. 2012;119(7):138898. https://doi.org/10.1016/j.ophtha.2012.03.053.

9. Schmidt-Erfurth U, Waldstein SM, Deak GG, Kundi M, Simader C. Pigment epithelial detachment followed by retinal cystoid degeneration leads to vision loss in treatment of neovascular age-related macular degeneration. Ophthalmology. 2015;122(4):822-32. https://doi.org/10.1016/j.ophtha.2 014.11.017.

10. Dirani A, Ambresin A, Marchionno L, Decugis D, Mantel I. Factors influencing the treatment response of pigment epithelium detachment in age-related macular degeneration. Am J Ophthalmol. 2015;160(4):732-8.e2.

11. Sato T, Suzuki M, Ooto S, Spaide RF. Multimodal imaging findings and multimodal vision testing in neovascular age-related macular degeneration. Retina. 2015;35(7):1292-302.

12. Guymer RH, Markey CM, McAllister IL, Gillies MC, Hunyor AP, Arnold JJ. Tolerating subretinal fluid in Neovascular age-related macular degeneration treated with Ranibizumab using a treat-and-extend regimen: fluid study 24month results. Ophthalmology. 2019;126(5):723-34. https://doi.org/10.1016/j. ophtha.2018.11.025

13. Hörster R, Ristau T, Sadda SR, Liakopoulos S. Individual recurrence intervals after anti-VEGF therapy for age-related macular degeneration. Graefes Arch Clin Exp Ophthalmol. 2011;249(5):645-52. https://doi.org/10.1007/s00417-01 $0-1588-2$.

14. Ying GS, Huang J, Maguire MG, Jaffe GJ, Grunwald JE, Toth C, et al. Baseline predictors for one-year visual outcomes with ranibizumab or bevacizumab for neovascular age-related macular degeneration. Ophthalmology. 2013; 120(1):122-9. https://doi.org/10.1016/j.ophtha.2012.07.042. 
15. Kim KT, Lee H, Kim JY, Lee S, Chae JB, Kim DY. Long-term visual/anatomic outcome in patients with fovea-involving fibrovascular pigment epithelium detachment presenting choroidal neovascularization on optical coherence tomography angiography. J Clin Med. 2020;9(6):1863. https://doi.org/10.33 90/jcm9061863.

16. Freund KB, Zweifel SA, Engelbert M. Do we need a new classification for choroidal neovascularization in age-related macular degeneration? Retina. 2010;30(9):1333-49.

17. Nakano Y, Kataoka K, Takeuchi J, Fujita A, Kaneko H, Shimizu H, et al. Vascular maturity of type 1 and type 2 choroidal neovascularization evaluated by optical coherence tomography angiography. PLoS One. 2019; 14(4):e0216304. https://doi.org/10.1371/journal.pone.0216304.

18. Arnold JJ, Markey CM, Kurstjens NP, Guymer RH. The role of sub-retinal fluid in determining treatment outcomes in patients with neovascular agerelated macular degeneration--a phase IV randomised clinical trial with ranibizumab: the FLUID study. BMC Ophthalmol. 2016;16(1):31. https://doi. org/10.1186/s12886-016-0207-3.

19. Kim KT, Lee H, Kim JY, Chae JB, Hyung S, Kim DY. Association between early anatomic response and intraocular pressure change after intravitreal dexamethasone implant: an optical coherence tomography study. J Clin Med. 2020;9(9):2692. https://doi.org/10.3390/jcm9092692.

20. Schuman SG, Koreishi AF, Farsiu S, Jung SH, Izatt JA, Toth CA. Photoreceptor layer thinning over drusen in eyes with age-related macular degeneration imaged in vivo with spectral-domain optical coherence tomography. Ophthalmology. 2009;116(3):488-96.e2.

21. Odrobina D, Laudańska-Olszewska I, Gozdek P, Maroszyński M, Amon M. Morphologic changes in the foveal photoreceptor layer before and after laser treatment in acute and chronic central serous chorioretinopathy documented in spectral-domain optical coherence tomography. J Ophthalmol. 2013;2013:361513.

22. Hoerster R, Muether PS, Sitnilska V, Kirchhof B, Fauser S. Fibrovascular pigment epithelial detachment is a risk factor for long-term visual decay in neovascular age-related macular degeneretion. Retina. 2014;34(9):1767-73.

23. Golbaz I, Ahlers C, Stock G, Schütze C, Schriefl S, Schlanitz F, et al. Quantification of the therapeutic response of intraretinal, subretinal, and subpigment epithelial compartments in exudative AMD during anti-VEGF therapy. Invest Ophthalmol Vis Sci. 2011;52(3):1599-605. https://doi.org/1 0.1167/iovs.09-5018.

24. Sulzbacher F, Kiss C, Kaider A, Eisenkoelbl S, Munk M, Roberts P, et al. Correlation of SD-OCT features and retinal sensitivity in neovascular agerelated macular degeneration. Invest Ophthalmol Vis Sci. 2012;53(10):644855. https://doi.org/10.1167/iovs.11-9162.

25. Jaffe GJ, Martin DF, Toth CA, Daniel E, Maguire MG, Ying GS, et al. Macular morphology and visual acuity in the comparison of age-related macular degeneration treatments trials. Ophthalmology. 2013;120(9):1860-70. https://doi.org/10.1016/j.ophtha.2013.01.073.

26. Schmidt-Erfurth $U$, Waldstein SM. A paradigm shift in imaging biomarkers in neovascular age-related macular degeneration. Prog Retin Eye Res. 2016;50: 1-24. https://doi.org/10.1016/j.preteyeres.2015.07.007.

27. Ritter M, Simader C, Bolz M, Deák GG, Mayr-Sponer U, Sayegh R, et al. Intraretinal cysts are the most relevant prognostic biomarker in neovascular age-related macular degeneration independent of the therapeutic strategy. Br J Ophthalmol. 2014;98(12):1629-35. https://doi.org/10.1136/bjophtha Imol-2014-305186.

28. Simader C, Ritter M, Bolz M, Deák GG, Mayr-Sponer U, Golbaz I, et al. Morphologic parameters relevant for visual outcome during anti-angiogenic therapy of neovascular age-related macular degeneration. Ophthalmology. 2014;121(6):1237-45. https://doi.org/10.1016/j.ophtha.2013.12.029.

29. Bolz M, Simader C, Ritter M, Ahlers C, Benesch T, Prünte C, et al. Morphological and functional analysis of the loading regimen with intravitreal ranibizumab in neovascular age-related macular degeneration. $\mathrm{Br}$ J Ophthalmol. 2010;94(2):185-9. https://doi.org/10.1136/bjo.2008.143974.

30. Heier JS, Brown DM, Chong V, Korobelnik JF, Kaiser PK, Nguyen QD, et al. Intravitreal aflibercept (VEGF trap-eye) in wet age-related macular degeneration. Ophthalmology. 2012;119(12):2537-48. https://doi.org/10.101 6/j.ophtha.2012.09.006

31. Chakravarthy U, Harding SP, Rogers CA, Downes SM, Lotery AJ, Culliford LA, et al. Alternative treatments to inhibit VEGF in age-related choroidal neovascularisation: 2-year findings of the IVAN randomised controlled trial. Lancet. 2013;382(9900):1258-67. https://doi.org/10.1016/S0140-6736(13)61 501-9.
32. Spaide R. Ranibizumab according to need: a treatment for age-related macular degeneration. Am J Ophthalmol. 2007;143(4):679-80. https://doi. org/10.1016/j.ajo.2007.02.024.

33. Berg K, Hadzalic E, Gjertsen I, Forsaa V, Berger LH, Kinge B, et al. Ranibizumab or bevacizumab for Neovascular age-related macular degeneration according to the Lucentis compared to Avastin study treatand-extend protocol: two-year results. Ophthalmology. 2016;123(1):51-9. https://doi.org/10.1016/j.ophtha.2015.09.018.

34. Barthelmes D, Nguyen V, Daien V, Campain A, Walton R, Guymer R, et al. Two year outcomes of "treat and extend" intravitreal therapy using aflibercept preferentially for neovascular age-related macular degeneration. Retina. 2018;38(1):20-8.

35. Silva R, Berta A, Larsen M, Macfadden W, Feller C, Monés J. Treat-and-extend versus monthly regimen in neovascular age-related macular degeneration: results with Ranibizumab from the trend study. Ophthalmology. 2018;125(1): 57-65. https://doi.org/10.1016/j.ophtha.2017.07.014.

\section{Publisher's Note}

Springer Nature remains neutral with regard to jurisdictional claims in published maps and institutional affiliations.

\section{Ready to submit your research? Choose BMC and benefit from:}

- fast, convenient online submission

- thorough peer review by experienced researchers in your field

- rapid publication on acceptance

- support for research data, including large and complex data types

- gold Open Access which fosters wider collaboration and increased citations

- maximum visibility for your research: over $100 \mathrm{M}$ website views per year

At BMC, research is always in progress.

Learn more biomedcentral.com/submissions 